\title{
Asymptotically J-Lacunary statistical equivalent of order $\alpha$ for sequences of sets
}

\author{
Ekrem Savaş \\ Istanbul Commerce University, Department of Mathematics, Sutluce-Istanbul, Turkey. \\ Communicated by Y. J. Cho
}

\begin{abstract}
This paper presents the following definition which is a natural combination of the definition for asymptotically equivalent of order $\alpha$, where $0<\alpha \leqslant 1$, J-statistically limit, and J-lacunary statistical convergence for sequences of sets. Let $(X, \rho)$ be a metric space and $\theta$ be a lacunary sequence. For any non-empty closed subsets $A_{k}, B_{k} \subseteq X$ such that $d\left(x, A_{k}\right)>0$ and $d\left(x, B_{k}\right)>0$ for each $x \in X$, we say that the sequences $\left\{A_{k}\right\}$ and $\left\{B_{k}\right\}$ are Wijsman asymptotically J-lacunary statistical equivalent of order $\alpha$ to multiple $L$, where $0<\alpha \leqslant 1$, provided that for each $\varepsilon>0$ and each $x \in X$,
\end{abstract}

$$
\left\{r \in \mathbb{N}: \frac{1}{h_{r}^{\alpha}}\left|\left\{k \in I_{r}:\left|d\left(x ; A_{k}, B_{k}\right)-L\right| \geqslant \epsilon\right\}\right| \geqslant \delta\right\} \in \mathcal{J},
$$

(denoted by $\left.\left\{A_{k}\right\} \stackrel{S_{\theta}^{L}\left(\mathcal{J}_{W}\right)^{\alpha}}{\sim}\left\{B_{k}\right\}\right)$ and simply asymptotically J-lacunary statistical equivalent of order $\alpha$ if $L=1$. In addition, we shall also present some inclusion theorems. The study leaves some interesting open problems. (C)2017 All rights reserved.

Keywords: Asymptotical equivalent, sequences of sets, ideal convergence, Wijsman convergence, J-statistical convergence, J-lacunary statistical convergence, statistical convergence of order $\alpha$. 2010 MSC: 40A35, 46A45.

\section{Introduction}

The concept of statistical convergence was introduce by Fast [6] in 1951. A sequence $\left(x_{k}\right)$ of real numbers is said to be statistically convergent to L if for arbitrary $\epsilon>0$,

$$
\frac{1}{\mathrm{n}}\left|\left\{\mathrm{k}<\mathrm{n}:\left|\mathrm{x}_{\mathrm{k}}-\mathrm{L}\right| \geqslant \epsilon\right\}\right|=0,
$$

where by $k<n$ we mean that $k=0,1,2, \cdots, n$ and the vertical bars indicate the number of elements in the enclosed set. In this case we write $s t-\lim x=\mathrm{L}$ or $x_{k} \mapsto \mathrm{L}(\mathrm{st})$.

By a lacunary $\theta=\left(k_{r}\right), r=0,1,2, \cdots$ where $k_{0}=0$, we shall mean an increasing sequence of nonnegative integers with $k_{r}-k_{r-1} \rightarrow \infty$ as $r \rightarrow \infty$. The intervals determined by $\theta$ will be denoted by

Email address: ekremsavas@yahoo.com (Ekrem Savaş)

doi:10.22436/jnsa.010.06.01 
$I_{r}=\left(k_{r-1}, k_{r}\right]$ and $h_{r}=k_{r}-k_{r-1}$. The ratio $\frac{k_{r}}{k_{r-1}}$ will be denoted by $q_{r}$. Moreover, the following concept is due to Fridy and Orhan [8].

A sequence $\left(x_{k}\right)$ of real numbers is said to be lacunary statistically convergent to $L$ (or, $S_{\theta}$-convergent to $L)$, if for any $\epsilon>0$,

$$
\lim _{r \rightarrow \infty} \frac{1}{h_{r}}\left|\left\{k \in I_{r}:\left|x_{k}-L\right| \geqslant \epsilon\right\}\right|=0,
$$

where $|A|$ denotes the cardinality of $A \subset \mathbb{N}$.

Recently in ([5] and [19]), we used ideals to introduce the concepts of J-statistical convergence and Jlacunary statistical convergence which naturally extend the notions of the above mentioned convergence. On the other hand, in [2] a different direction was given to the study of statistical convergence where the notion of statistical convergence of order $\alpha, 0<\alpha \leqslant 1$ was introduced by replacing $n$ by $n^{\alpha}$ in the denominator in the definition of statistical convergence. One can also see [3, 15] for related works. In 1993 Marouf [12] presented definitions for asymptotically equivalent sequences and asymptotic regular matrices. Also, in 1997, Li [11] presented and studied asymptotic equivalence of sequences and summability. In 2003, Patterson [14] extended these concepts by presenting an asymptotically statistical equivalent analog of these definitions and natural regularity conditions for non-negative summability matrices.

The idea of statistical convergence was further extended to J-convergence in [10] using the notion of ideals of $\mathbb{N}$ with many interesting consequences. More investigations in this direction and more applications of ideals can be found in [4,5,16-20] where many important references can be found.

In present paper, we use asymptotical equivalent of set sequences to introduce the concept Wijsman asymptotically J-statistical equivalent of order $\alpha$ and Wijsman asymptotically $\mathcal{J}$-lacunary statistical equivalent of order $\alpha$ for sequences of set. In addition to these definitions, natural inclusion theorems shall also be presented.

\section{Definitions and preliminaries}

The following definitions and notions will be needed in the sequel.

Definition 2.1 ([12]). Two non-negative sequences $x=\left(x_{k}\right)$ and $y=\left(y_{k}\right)$ are said to be asymptotically equivalent, if

$$
\lim _{k} \frac{x_{k}}{y_{k}}=1
$$

(denoted by $x \sim y$ ).

Definition $2.2([7])$. The sequence $x=\left(x_{k}\right)$ has statistic limit $L$, denoted by $s t-\lim x_{k}=L$, provided that for every $\epsilon>0$,

$$
\lim _{n} \frac{1}{n}\left\{\text { the number of } k \leqslant n:\left|x_{k}-L\right| \geqslant \epsilon\right\}=0 .
$$

The next definition is natural combination of Definitions 2.1 and 2.2.

Definition 2.3 ([14]). Two non-negative sequences $x=\left(x_{k}\right)$ and $y=\left(y_{k}\right)$ are said to be asymptotically statistical equivalent of multiple L provided that for every $\epsilon>0$,

$$
\lim _{n} \frac{1}{n}\left\{\text { the number of } k<n:\left|\frac{x_{k}}{y_{k}}-L\right| \geqslant \epsilon\right\}=0 \text {, }
$$

(denoted by $x \stackrel{S_{L}}{\sim} y$ ), and simply asymptotically statistical equivalent if $L=1$.

Definition 2.4. A family $\mathcal{J} \subset 2^{\mathbb{N}}$ is said to be an ideal of $\mathbb{N}$, if the following conditions hold:

(a) $A, B \in \mathcal{J}$ implies $A \cup B \in \mathcal{J}$; 
(b) $A \in \mathcal{J}, B \subset A$ implies $B \in \mathcal{J}$.

Definition 2.5. A non-empty family $F \subset 2^{\mathbb{N}}$ is said to be a filter of $\mathbb{N}$, if the following conditions hold:

(a) $\phi \notin F$;

(b) $A, B \in F$ implies $A \cap B \in F$;

(c) $A \in F, A \subset B$ implies $B \in F$.

If $\mathcal{J}$ is a proper ideal of $\mathbb{N}$ (i.e., $\mathbb{N} \notin \mathcal{J}$ ), then the family of sets $F(\mathcal{J})=\{M \subset \mathbb{N}: \exists A \in \mathcal{J}: M=\mathbb{N} \backslash A\}$ is a filter of $\mathbb{N}$. It is called the filter associated with the ideal.

Definition 2.6. A proper ideal $\mathcal{J}$ is said to be admissible if $\{n\} \in \mathcal{J}$ for each $n \in \mathbb{N}$.

Throughout $\mathcal{J}$ will stand for a proper admissible ideal of $\mathbb{N}$.

Definition 2.7 ([10]). Let $\mathcal{J} \subset 2^{\mathbb{N}}$ be a proper admissible ideal in $\mathbb{N}$. Then the sequence $\left(x_{k}\right)$ of elements of $\mathbb{R}$ is said to be J J-convergent to $L \in \mathbb{R}$, if for each $\epsilon>0$ the set $A(\epsilon)=\left\{k \in \mathbb{N}:\left|x_{k}-L\right| \geqslant \epsilon\right\} \in \mathcal{J}$.

Let $(X, \rho)$ be a metric space. For any point $x \in X$ and any non-empty subset $A$ of $X$, we define the distance from $x$ to $A$ by

$$
d(x, A)=\inf _{a \in A} \rho(x, A) .
$$

Definition $2.8([1])$. Let $(X, \rho)$ be a metric space. For any non-empty closed subsets $A, A_{k} \subseteq X$, we say that the sequence $\left\{A_{k}\right\}$ is Wijsman convergent to $A$, if

$$
\lim _{k \rightarrow \infty} d\left(x, A_{k}\right)=d(x, A),
$$

for each $x \in X$. In this case we write $W-\lim A_{k}=A$.

In [13], statistical convergence of sequences of sets was given by Nuray and Rhoades as follows:

Definition 2.9. Let $(X, \rho)$ be a metric space. For any non-empty closed subsets $A, A_{k} \subseteq X$, we say that the sequence $\left\{A_{k}\right\}$ is Wijsman statistical convergent to $A$, if for $\varepsilon>0$ and for each $x \in X$,

$$
\lim _{n \rightarrow \infty} \frac{1}{n}\left|\left\{k \leqslant n:\left|d\left(x, A_{k}\right)-d(x, A)\right| \geqslant \varepsilon\right\}\right|=0 .
$$

In this case we write st-lim $A_{k}=A$ or $A_{k} \rightarrow A(W S)$.

We now have

Definition $2.10([9,18])$. Let $(X, \rho)$ be a metric space and $\theta$ be lacunary sequence. For any non-empty closed subsets $A, A_{k} \subset X$, we say that the sequence $\left\{A_{k}\right\}$ is Wijsman J-lacunary statistical convergent to $A$ or $S_{\theta}\left(J_{W}\right)$-convergent to $A$, if for each $\varepsilon>0, \delta>0$ and for each $x \in X$,

$$
\left\{r \in \mathbb{N}: \frac{1}{h_{r}}\left|\left\{k \in I_{r}:\left|d\left(x, A_{k}\right)-d(x, A)\right| \geqslant \varepsilon\right\}\right| \geqslant \delta\right\} \in \mathcal{J} .
$$

In this case, we write $A_{k} \rightarrow A\left(S_{\theta}\left(\mathcal{J}_{W}\right)\right)$.

\section{Main results}

In this section we shall give some new definitions and also examine some inclusion relations.

Definition $3.1([18])$. Let $(X, \rho)$ be a metric space. For any non-empty closed subsets $A, A_{k} \subset X$, we say that the sequence $\left\{A_{k}\right\}$ is Wijsman J-statistical convergent of order $\alpha$ to $A$ or $S\left(J_{W}\right)$-convergent of order $\alpha$ $(0<\alpha \leqslant 1)$ to $A$, if for each $\varepsilon>0, \delta>0$ and for each $x \in X$,

$$
\left\{n \in \mathbb{N}: \frac{1}{n^{\alpha}}\left|\left\{k \leqslant n:\left|d\left(x, A_{k}\right)-d(x, A)\right| \geqslant \epsilon\right\}\right| \geqslant \delta\right\} \in \mathcal{J} .
$$

In this case we write $A_{k} \rightarrow A\left(S\left(\mathcal{J}_{\mathcal{W}}\right)^{\alpha}\right)$. The class of all Wijsman J-statistical sequences of order $\alpha$ will be 
denoted by simply $S\left(\mathcal{J}_{\mathcal{W}}\right)^{\alpha}$.

Let $(X, \rho)$ be a metric space. For any non-empty closed subsets $A_{k}, B_{k} \subseteq X$, we define $d\left(x ; A_{k}, B_{k}\right)$ as follows:

$$
d\left(x ; A_{k}, B_{k}\right)= \begin{cases}\frac{d\left(x, A_{k}\right)}{d\left(x, B_{k}\right)}, & x \notin A_{k} \cup B_{k}, \\ L, & x \in A_{k} \cup B_{k} .\end{cases}
$$

The next definition is natural combination of Definitions 2.1 and 3.1.

Definition 3.2. Let $(X, \rho)$ be a metric space. For any non-empty closed subsets $A_{k}, B_{k} \subseteq X$ such that $d\left(x, A_{k}\right)>0$ and $d\left(x, B_{k}\right)>0$ for each $x \in X$, we say that the sequences $\left\{A_{k}\right\}$ and $\left\{B_{k}\right\}$ are Wijsman asymptotically J-statistical equivalent of order $\alpha(0<\alpha \leqslant 1)$ to multiple L provided that for each $\epsilon>0$, $\delta>0$ and each $x \in X$,

$$
\left\{n \in \mathbb{N}: \frac{1}{n^{\alpha}}\left|\left\{k \leqslant n:\left|d\left(x ; A_{k}, B_{k}\right)-L\right| \geqslant \epsilon\right\}\right| \geqslant \delta\right\} \in \mathcal{J},
$$

(denoted by $\left\{A_{k}\right\} \stackrel{S^{L}\left(\mathcal{J}_{\mathcal{W}}\right)^{\alpha}}{\sim}\left\{B_{k}\right\}$ ) and simply Wijsman asymptotically J-statistical equivalent of order $\alpha$ if $L=1$. Furthermore, let $S^{L}\left(\mathcal{J}_{W}\right)^{\alpha}$ denote the set of $\left\{A_{k}\right\}$ and $\left\{B_{k}\right\}$ such that $\left\{A_{k}\right\} \stackrel{S^{L}\left(\mathcal{J}_{\mathcal{W}}\right)^{\alpha}}{\sim}\left\{B_{k}\right\}$.

Remark 3.3. If $\mathcal{J}=\mathcal{J}_{\text {fin }}=\{\mathrm{A} \subseteq \mathbf{N}: A$ is a finite subset $\}$, Wijsman asymptotically $\mathcal{J}$-statistical equivalent of order $\alpha$ to multiple L coincides with Wijsman asymptotically statistical equivalent of order $\alpha$ to multiple L. For an arbitrary ideal $\mathcal{J}$ and for $\alpha=1$ it coincides with Wijsman asymptotically J-statistical equivalent of multiple L. When $\mathcal{J}=\mathcal{J}_{\text {fin }}$ and $\alpha=1$ it becomes only Wijsman asymptotically statistical equivalent of multiple L for set sequences, [22, 23].

Definition 3.4 ([18]). Let $(X, \rho)$ be a metric space and $\theta$ be a lacunary sequence. For any non-empty closed subsets $A, A_{k} \subset X$, we say that the sequence $\left\{A_{k}\right\}$ is Wijsman J-lacunary statistical convergent of order $\alpha$ to $A$ or $S_{\theta}\left(J_{W}\right)$-convergent of order $\alpha(0<\alpha \leqslant 1)$ to $A$, if for each $\varepsilon>0, \delta>0$ and for each $x \in X$,

$$
\left\{r \in \mathbb{N}: \frac{1}{h_{r}^{\alpha}}\left|\left\{k \in I_{r}:\left|d\left(x, A_{k}\right)-d(x, A)\right| \geqslant \epsilon\right\}\right| \geqslant \delta\right\} \in \mathcal{J} .
$$

In this case we write $A_{k} \rightarrow A\left(S_{\theta}\left(\mathcal{J}_{\mathcal{W}}\right)^{\alpha}\right)$. The class of all J-lacunary statistically convergent sequences of order $\alpha$ will be denoted by $S_{\theta}\left(\mathcal{J}_{\mathcal{W}}\right)^{\alpha}$.

Remark 3.5. If $\mathcal{J}=\mathcal{J}_{\text {fin }}=\{A \subseteq \mathbf{N}: A$ is a finite subset $\}$, Wijsman J-lacunary statistical convergent of order $\alpha$ coincides with Wijsman lacunary statistical convergent of order $\alpha$. For an arbitrary ideal J and for $\alpha=1$ it coincides with Wijsman $\mathcal{J}$-lacunary statistical convergent, [18]. When $\mathcal{J}=\mathcal{J}_{\text {fin }}$ and $\alpha=1$ it becomes only Wijsman lacunary statistical convergent for set sequences, [21].

The next definition is natural combination of Definitions 2.1 and 3.4.

Definition 3.6. Let $(X, \rho)$ be a metric space and $\theta$ be a lacunary sequence. For any non-empty closed subsets $A_{k}, B_{k} \subseteq X$ such that $d\left(x, A_{k}\right)>0$ and $d\left(x, B_{k}\right)>0$ for each $x \in X$, we say that the sequences $\left\{A_{k}\right\}$ and $\left\{B_{k}\right\}$ are Wijsman asymptotically J-lacunary statistical equivalent of order $\alpha(0<\alpha \leqslant 1)$ to multiple $L$ provided that for each $\varepsilon>0, \delta>0$ and each $x \in X$,

$$
\left\{r \in \mathbb{N}: \frac{1}{h_{r}^{\alpha}}\left|\left\{k \in I_{r}:\left|d\left(x ; A_{k}, B_{k}\right)-L\right| \geqslant \epsilon\right\}\right| \geqslant \delta\right\} \in \mathcal{J},
$$

(denoted by $\left\{A_{k}\right\} \stackrel{S_{\theta}^{L}(\mathcal{J})^{\alpha}}{\sim}\left\{B_{k}\right\}$ ) and simply asymptotically J-lacunary statistical equivalent of order $\alpha$ if $L=1$. Furthermore, let $S_{\theta}^{L}\left(\mathcal{J}_{\mathcal{W}}\right)^{\alpha}$ denote the set of $\left\{A_{k}\right\}$ and $\left\{B_{k}\right\}$ such that $\left\{A_{k}\right\} \stackrel{S_{\theta}^{L}\left(\mathcal{J}_{\mathcal{W}}\right)^{\alpha}}{\sim}\left\{B_{k}\right\}$. 
Remark 3.7. For $\alpha=1$, the above definition coincides with Wijsman asymptotically J-lacunary statistical equivalent of multiple $\mathrm{L}$, (see, $[9,23]$ ). If we take $\mathcal{J}=\mathcal{J}_{\text {fin }}$ and $\alpha=1$ Wijsman asymptotically lacunary statistical equivalent of multiple $L$ is a special case of Wijsman asymptotically J-lacunary statistical equivalent of order $\alpha$ to multiple L, (see, [22]) .

Theorem 3.8. Let $0<\alpha \leqslant \beta \leqslant 1$. Then $\mathrm{S}\left(\mathcal{J}_{W}\right)^{\alpha} \subset \mathrm{S}\left(\mathcal{J}_{W}\right)^{\beta}$.

Proof. Let $0<\alpha \leqslant \beta \leqslant 1$. Then

$$
\frac{\left|\left\{k \leqslant n:\left|d\left(x ; A_{k}, B_{k}\right)-L\right| \geqslant \epsilon\right\}\right|}{n^{\beta}} \leqslant \frac{\left|\left\{k \leqslant n:\left|d\left(x ; A_{k}, B_{k}\right)-L\right| \geqslant \epsilon\right\}\right|}{n^{\alpha}},
$$

and so for any $\delta>0$,

$$
\left\{n \in \mathbb{N}: \frac{\left|\left\{k \leqslant n:\left|d\left(x ; A_{k}, B_{k}\right)-L\right| \geqslant \epsilon\right\}\right|}{n^{\beta}} \geqslant \delta\right\} \subset\left\{n \in \mathbb{N}: \frac{\left|\left\{k \leqslant n:\left|d\left(x ; A_{k}, B_{k}\right)-L\right| \geqslant \epsilon\right\}\right|}{n^{\alpha}} \geqslant \delta\right\} .
$$

Hence if the set on the right hand side belongs to the ideal $\mathcal{J}$ then obviously the set on the left hand side also belongs to $\mathcal{J}$. This shows that $S\left(\mathcal{J}_{\mathcal{W}}\right)^{\alpha} \subset S\left(\mathcal{J}_{\mathcal{W}}\right)^{\beta}$.

Similarly we can show that

Theorem 3.9. Let $0<\alpha \leqslant \beta \leqslant 1$. Then

(i) $S_{\theta}^{\mathrm{L}}\left(\mathcal{J}_{\mathcal{W}}\right)^{\alpha} \subset S_{\theta}^{\mathrm{L}}\left(\mathcal{J}_{\mathcal{W}}\right)^{\beta}$.

(ii) In particular $\mathrm{S}_{\theta}^{\mathrm{L}}\left(\mathcal{J}_{\mathcal{W}}\right)^{\alpha} \subset S_{\theta}^{\mathrm{L}}\left(\mathcal{J}_{\mathcal{W}}\right)$.

Definition 3.10. Let $(X, \rho)$ be a metric space and $\theta$ be lacunary sequence. For any non-empty closed subsets $A_{k}, B_{k} \subseteq X$ such that $d\left(x, A_{k}\right)>0$ and $d\left(x, B_{k}\right)>0$ for each $x \in X$, we say that the sequences $\left\{A_{k}\right\}$ and $\left\{B_{k}\right\}$ are strongly Wijsman asymptotically J-lacunary equivalent of order $\alpha(0<\alpha \leqslant 1)$ to multiple $L$ provided that for each $\varepsilon>0$ and each $x \in X$,

$$
\left\{r \in \mathbb{N}: \frac{1}{h_{r}^{\alpha}} \sum_{k \in I_{r}}\left|d\left(x ; A_{k}, B_{k}\right)-L\right| \geqslant \epsilon\right\} \in \mathcal{J},
$$

(denoted by $A_{k} \stackrel{N_{\theta}^{L}\left(\mathcal{J}_{\mathcal{W}}\right)^{\alpha}}{\sim} B_{k}$ ) and simply strong asymptotically J-lacunary statistical equivalent of order $\alpha$ if $L=1$. Further, let $N_{\theta}^{L}\left(\mathcal{J}_{\mathcal{W}}\right)^{\alpha}$ denote the set of $A_{k}$ and $B_{k}$ such that $\left\{A_{k}\right\} \stackrel{N_{\theta}^{L}\left(\mathcal{J}_{\mathcal{W}}\right)^{\alpha}}{\sim}\left\{B_{k}\right\}$.

We prove the following

Theorem 3.11. Let $\theta$ be a lacunary sequence, if $\left\{A_{k}\right\} \stackrel{N_{\theta}^{L}\left(\mathcal{J}_{\mathcal{W}}\right)^{\alpha}}{\sim}\left\{B_{k}\right\}$ then $\left\{A_{k}\right\} \stackrel{S_{\theta}^{L}\left(\mathcal{J}_{\mathcal{W}}\right)^{\alpha}}{\sim}\left\{B_{k}\right\}$.

Proof. If $\epsilon>0$ and $\left\{A_{k}\right\} \stackrel{N_{\theta}^{L}(\mathcal{J})^{\alpha}}{\sim}\left\{B_{k}\right\}$, we can write

$$
\sum_{k \in I_{r}}\left|d\left(x ; A_{k}, B_{k}\right)-L\right| \geqslant \sum_{k \in I_{r},\left|d\left(x ; A_{k}, B_{k}\right)-L\right| \geqslant \epsilon}\left|d\left(x ; A_{k}, B_{k}\right)-L\right| \geqslant \epsilon\left|\left\{k \in I_{r}:\left|d\left(x ; A_{k}, B_{k}\right)-L\right| \geqslant \epsilon\right\}\right|,
$$

and so

$$
\frac{1}{\epsilon \cdot h_{r}^{\alpha}} \sum_{k \in I_{r}}\left|d\left(x ; A_{k}, B_{k}\right)-L\right| \geqslant \frac{1}{h_{r}^{\alpha}}\left|\left\{k \in I_{r}:\left|d\left(x ; A_{k}, B_{k}\right)-L\right| \geqslant \epsilon\right\}\right| .
$$

Then for any $\delta>0$

$$
\left\{r \in \mathbb{N}: \frac{1}{h_{r}^{\alpha}}\left|\left\{k \in I_{r}:\left|d\left(x ; A_{k}, B_{k}\right)-L\right| \geqslant \epsilon\right\}\right| \geqslant \delta\right\} \subseteq\left\{r \in \mathbb{N}: \frac{1}{h_{r}^{\alpha}} \sum_{k \in I_{r}}\left|d\left(x ; A_{k}, B_{k}\right)-L\right| \geqslant \epsilon . \delta\right\} \in \mathcal{J} .
$$

This proves the result. 
Remark 3.12. In [23, Theorem 1] it was further proved that

(i) $x \in l_{\infty}$, the set of the bounded sequences and $\left\{A_{k}\right\} \stackrel{S_{\theta}^{L}\left(\mathcal{J}_{\mathcal{W}}\right)}{\sim}\left\{B_{k}\right\} \Rightarrow x \stackrel{N_{\theta}^{L}\left(\mathcal{J}_{\mathcal{W}}\right)}{\sim} y$;

(ii) $S_{\theta}^{\mathrm{L}}\left(\mathcal{J}_{\mathcal{W}}\right) \cap l_{\infty}=N_{\theta}^{\mathrm{L}}\left(\mathcal{J}_{\mathcal{W}}\right) \cap l_{\infty}$.

However whether these results remain true for $0<\alpha<1$ is not clear and we leave them as open problems.

We now investigate the relationship between $\left\{A_{k}\right\} \stackrel{S^{L}\left(\mathcal{J}_{\mathcal{W}}\right)^{\alpha}}{\sim}\left\{B_{k}\right\}$ and $\left\{A_{k}\right\} \stackrel{S_{\theta}^{L}\left(\mathcal{J}_{\mathcal{W}}\right)^{\alpha}}{\sim}\left\{B_{k}\right\}$.

Theorem 3.13. Let $\theta$ be a lacunary sequence, then

$$
\left\{\mathrm{A}_{\mathrm{k}}\right\} \stackrel{\mathrm{S}^{\mathrm{L}}\left(\mathcal{J}_{\mathcal{W}}\right)^{\alpha}}{\sim}\left\{\mathrm{B}_{\mathrm{k}}\right\} \text { implies }\left\{\mathrm{A}_{\mathrm{k}}\right\} \stackrel{S_{\mathrm{\theta}}^{\mathrm{L}}\left(\mathcal{J}_{\mathcal{W}}\right)^{\alpha}}{\sim}\left\{\mathrm{B}_{\mathrm{k}}\right\},
$$

if $\liminf q_{r}^{\alpha}>1$.

Proof. Suppose first that $\lim \underset{r}{\inf } q_{r}^{\alpha}>1$. Then there exists $\sigma>0$ such that $q_{r}^{\alpha} \geqslant 1+\sigma$ for sufficiently large $r$ which implies that

$$
\frac{h_{r}^{\alpha}}{k_{r}^{\alpha}} \geqslant \frac{\sigma}{1+\sigma}
$$

Since $x \stackrel{S^{L}\left(\mathcal{J}_{\mathcal{W}}\right)^{\alpha}}{\sim}$, then for every $\epsilon>0$ and for sufficiently large $r$, we have

$$
\begin{aligned}
\frac{1}{k_{r}^{\alpha}}\left|\left\{k \leqslant k_{r}:\left|d\left(x ; A_{k}, B_{k}\right)-L\right| \geqslant \epsilon\right\}\right| & \geqslant \frac{1}{k_{r}^{\alpha}}\left|\left\{k \in I_{r}:\left|d\left(x ; A_{k}, B_{k}\right)-L\right| \geqslant \epsilon\right\}\right| \\
& \geqslant \frac{\sigma}{1+\sigma} \cdot \frac{1}{h_{r}^{\alpha}}\left|\left\{k \in I_{r}:\left|d\left(x ; A_{k}, B_{k}\right)-L\right| \geqslant \epsilon\right\}\right| .
\end{aligned}
$$

Then for any $\delta>0$, we get

$$
\begin{aligned}
\{r \in \mathbb{N}: & \left.\frac{1}{h_{r}^{\alpha}}\left|\left\{k \in I_{r}:\left|d\left(x ; A_{k}, B_{k}\right)-L\right| \geqslant \epsilon\right\}\right| \geqslant \delta\right\} \\
& \subseteq\left\{r \in \mathbb{N}: \frac{1}{k_{r}^{\alpha}}\left|\left\{k \leqslant k_{r}:\left|d\left(x ; A_{k}, B_{k}\right)-L\right| \geqslant \epsilon\right\}\right| \geqslant \frac{\delta \sigma}{(1+\sigma)}\right\} \in \mathcal{J} .
\end{aligned}
$$

This proves the result.

Remark 3.14. The converse of this result is not clear for $\alpha<1$ and we leave it as an open problem.

For the next result we assume that the lacunary sequence $\theta$ satisfies the condition that for any set $\mathrm{C} \in \mathrm{F}(\mathcal{J}), \bigcup\left\{n: k_{r-1}<n<k_{r}, r \in C\right\} \in F(\mathcal{J})$.

Theorem 3.15. For a lacunary sequence $\theta$ satisfying the above condition,

$$
\left\{\mathrm{A}_{\mathrm{k}}\right\} \stackrel{\mathrm{S}_{\theta}^{\mathrm{L}}\left(\mathcal{J}_{\mathcal{W}}\right)^{\alpha}}{\sim}\left\{\mathrm{B}_{\mathrm{k}}\right\} \text { implies }\left\{\mathrm{A}_{\mathrm{k}}\right\} \stackrel{\mathrm{S}^{\mathrm{L}}\left(\mathcal{J}_{\mathcal{W}}\right)^{\alpha}}{\sim}\left\{\mathrm{B}_{\mathrm{k}}\right\},
$$

if $B:=\sup _{r} \sum_{i=0}^{r-1} \frac{h_{i+1}^{\alpha}}{\left(k_{r-1}\right)^{\alpha}}<\infty$.

Proof. Suppose that $\left\{A_{k}\right\} \stackrel{S_{\theta}^{L}\left(\mathcal{J}_{\mathcal{W}}\right)^{\alpha}}{\sim}\left\{B_{k}\right\}$ and for $\epsilon, \delta, \delta_{1}>0$ define the sets

$$
C=\left\{r \in \mathbb{N}: \frac{1}{h_{r}{ }^{\alpha}}\left|\left\{k \in I_{r}:\left|d\left(x ; A_{k}, B_{k}\right)-L\right| \geqslant \epsilon\right\}\right|<\delta\right\},
$$

and

$$
\mathrm{T}=\left\{\mathrm{n} \in \mathbb{N}: \frac{1}{n^{\alpha}}\left|\left\{k \leqslant n:\left|\mathrm{d}\left(x ; A_{k}, B_{k}\right)-L\right| \geqslant \epsilon\right\}\right|<\delta_{1}\right\} .
$$

It is obvious from our assumption that $C \in F(\mathcal{J})$, the filter associated with the ideal $\mathcal{J}$. Further observe that 


$$
A_{j}=\frac{1}{h_{j}{ }^{\alpha}}\left|\left\{k \in I_{j}:\left|d\left(x ; A_{k}, B_{k}\right)-L\right| \geqslant \epsilon\right\}\right|<\delta,
$$

for all $j \in C$. Let $n \in \mathbb{N}$ be such that $k_{r-1}<n<k_{r}$ for some $r \in C$. Now

$$
\begin{aligned}
\frac{1}{n^{\alpha}}\left|\left\{k \leqslant n:\left|d\left(x ; A_{k}, B_{k}\right)-L\right| \geqslant \epsilon\right\}\right| \leqslant & \frac{1}{k_{r-1}^{\alpha}}\left|\left\{k \leqslant k_{r}:\left|d\left(x ; A_{k}, B_{k}\right)-L\right| \geqslant \epsilon\right\}\right| \\
= & \frac{1}{k_{r-1}^{\alpha}}\left|\left\{k \in I_{1}:\left|d\left(x ; A_{k}, B_{k}\right)-L\right| \geqslant \epsilon\right\}\right|+\cdots \\
& +\frac{1}{k_{r-1}^{\alpha}}\left|\left\{k \in I_{r}:\left|d\left(x ; A_{k}, B_{k}\right)-L\right| \geqslant \epsilon\right\}\right| \\
= & \frac{k_{1}^{\alpha}}{k_{r-1}^{\alpha}} \frac{1}{h_{1}^{\alpha}}\left|\left\{k \in I_{1}:\left|d\left(x ; A_{k}, B_{k}\right)-L\right| \geqslant \epsilon\right\}\right| \\
& +\frac{\left(k_{2}-k_{1}\right)^{\alpha}}{k_{r-1}^{\alpha}} \frac{1}{h_{2}^{\alpha}}\left|\left\{k \in I_{2}:\left|d\left(x ; A_{k}, B_{k}\right)-L\right| \geqslant \epsilon\right\}\right|+\cdots \\
& +\frac{\left(k_{r}-k_{r-1}\right)^{\alpha}}{k_{r-1}^{\alpha}} \frac{1}{h_{r}^{\alpha}}\left|\left\{k \in I_{r}:\left|d\left(x ; A_{k}, B_{k}\right)-L\right| \geqslant \epsilon\right\}\right| \\
= & \frac{k_{1}^{\alpha}}{k_{r-1}^{\alpha}} A_{1}+\frac{\left(k_{2}-k_{1}\right)^{\alpha}}{k_{r-1}^{\alpha}} A_{2}+\cdots+\frac{\left(k_{r}-k_{r-1}\right)^{\alpha}}{k_{r-1}^{\alpha}} A_{r} \\
\leqslant & \sup _{j \in C} A_{j} \sup _{r}^{r} \sum_{i=0}^{r} \frac{\left(k_{i+1}-k_{i}\right)^{\alpha}}{k_{r-1}^{\alpha}}<B \delta .
\end{aligned}
$$

Choosing $\delta_{1}=\frac{\delta}{B}$ and in view of the fact that $\bigcup\left\{n: k_{r-1}<n<k_{r}, r \in C\right\} \subset T$ where $C \in F(\mathcal{J})$ it follows from our assumption on $\theta$ that the set $T$ also belongs to $F(\mathcal{J})$ and this completes the proof of the theorem.

\section{Acknowledgment}

The author wishes to thank the referees for their valuable suggestions which improved the presentation of the paper.

\section{References}

[1] M. Baronti, P. L. Papini, Convergence of sequences of sets, Methods of functional analysis in approximation theory, Bombay, (1985), Internat. Schriftenreihe Numer. Math., Birkhäuser, Basel, 76 (1986), 133-155. 2.8

[2] R. Çolak, Statistical convergence of order $\alpha$, Modern Methods in Analysis and Its Applications, Anamaya Pub., New Delhi, India, (2010), 121-129. 1

[3] R. Çolak, C. A. Bektaş, 入-statistical convergence of order $\alpha$, Acta Math. Sci. Ser. B Engl. Ed., 31 (2011), 953-959. 1

[4] P. Das, E. Savaş, On I-statistical and I-lacunary statistical convergence of order $\alpha$, Bull. Iranian Math. Soc., 40 (2014), 459-472. 1

[5] P. Das, E. Savaş, S. K. Ghosal, On generalizations of certain summability methods using ideals, Appl. Math. Lett., 24 (2011), 1509-1514. 1

[6] H. Fast, Sur la convergence statistique, (French) Colloquium Math., 2 (1951), 241-244. 1

[7] J. A. Fridy, On statistical convergence, Analysis, 5 (1985), 301-313. 2.2

[8] J. A. Fridy, C. Orhan, Lacunary statistical convergence, Pacific J. Math., 160 (1993), 43-51. 1

[9] Ö. Kişi, E. Savaş, F. Nuray, On I-asymptotically lacunary statistical equivalence of sequences of sets, (preprint). $2.10,3.7$

[10] P. Kostyrko, T. Śalát, W. Wilczyński, I-convergence, Real Anal. Exchange, 26 (2000/01), 669-685. 1, 2.7

[11] J.-L. Li, Asymptotic equivalence of sequences and summability, Internat. J. Math. Math. Sci., 20 (1997), 749-757. 1

[12] M. S. Marouf, Asymptotic equivalence and summability, Internat. J. Math. Math. Sci., 16 (1993), 755-762. 1, 2.1

[13] F. Nuray, B. E. Rhoades, Statistical convergence of sequences of sets, Fasc. Math., 49 (2012), 87-99. 2

[14] R. F. Patterson, On asymptotically statistical equivalent sequences, Demonstratio Math., 36 (2003), 149-153. 1, 2.3

[15] E. Savaş, Double almost lacunary statistical convergence of order $\alpha$, Adv. Difference Equ., 2013 (2013), 10 pages. 1 
[16] E. Savaş, On I-asymptotically lacunary statistical equivalent sequences, Adv. Difference Equ., 2013 (2013), 7 pages. 1

[17] E. Savaş, On asymptotically I-lacunary statistical equivalent sequences of order $\alpha$, International Conference on Pure Mathematics-Applied Mathematics, Venice, Italy, (2014), 15-17.

[18] E. Savaş, On I-lacunary statistical convergence of order $\alpha$ for sequences of sets, Filomat, 29 (2015), 1223-1229. 2.10, 3.1, $3.4,3.5$

[19] E. Savaş, P. Das, A generalized statistical convergence via ideals, Appl. Math. Lett., 24 (2011), 826-830. 1

[20] E. Savaş, H. Gumuş, A generalization on I-asymptotically lacunary statistical equivalent sequences, J. Inequal. Appl., 2013 (2013), 9 pages. 1

[21] U. Ulusu, F. Nuray, Lacunary statistical convergence of sequences of sets, Prog. Appl. Math., 4 (2012), 99-109. 3.5

[22] U. Ulusu, F. Nuray, On asymptotically lacunary statistical equivalent set sequences, J. Math., 2013 (2013), 5 pages. 3.3, 3.7

[23] U. Ulusu, E. Savaş, An extension of asymptotically lacunary statistical equivalence set sequences, J. Inequal. Appl., 2014 (2014), 8 pages. $3.3,3.7,3.12$ 